\title{
The I148M Variant of PNPLA3 Reduces the Response to Docosahexaenoic Acid in Children with Non-Alcoholic Fatty Liver Disease
}

\author{
Valerio Nobili, Giorgio Bedogni, ${ }^{2}$ Benedetta Donati, ${ }^{3}$ Anna Alisi, and Luca Valenti ${ }^{3}$ \\ ${ }^{1}$ Hepatometabolic Unit, Bambino Gesù Children's Hospital, Roma, Italy. \\ ${ }^{2}$ Clinical Epidemiology Unit, Liver Research Center, Trieste, Italy. \\ ${ }^{3}$ Department of Pathophysiology and Transplantation, Università degli Studi di Milano, \\ Internal Medicine, Fondazione Cà Granda IRCCS Ospedale Maggiore Policlinico, Milan, Italy.
}

\begin{abstract}
The aim of this secondary analysis of a randomized controlled trial was to test whether the I148M variant of Patatin-like phospholipase domain-containing protein-3 (PNPLA3) is associated with the response to docosahexaenoic acid (DHA) in children with non-alcoholic fatty liver disease (NAFLD). Sixty children with NAFLD were randomized in equal numbers to DHA $250 \mathrm{mg} /$ day, DHA $500 \mathrm{mg} /$ day or placebo. Coherently with the primary analysis, the probability of more severe steatosis after 24 months of DHA supplementation was 50\% lower [ $95 \%$ confidence interval (CI) $-59 \%$ to $-42 \%$ )] in the combined DHA 250 and $500 \mathrm{mg}$ /day groups versus placebo. The present secondary analysis revealed an independent effect of PNPLA3 status on the response to DHA. In fact, the probability of more severe steatosis was higher (37\%, 95\% CI 26$48 \%$ ) for the PNPLA3 M/M versus I/M genotype and lower (-12\%, 95\% CI $-21 \%$ to $-3 \%$ ) for the I/I versus I/M genotype (Somers' D for repeated measures). We conclude that the 148M allele of PNPLA3 is associated with lower response, and the 148I allele with greater response, to DHA supplementation in children with NAFLD.
\end{abstract}

KEY WORDS: • children • docosahexaenoic acid $\bullet$ non-alcoholic fatty liver disease $\bullet$ randomized controlled trial

$\mathbf{F}$ OLLOWING THE TRAIL of the obesity epidemics, nonalcoholic fatty liver disease (NAFLD) has become the major cause of pediatric chronic liver disease in industrialized countries, as it affects $3-10 \%$ of children, reaching approximately $70-80 \%$ in the presence of obesity. ${ }^{1}$ NAFLD encompasses a spectrum of liver damage ranging from simple fat accumulation to non-alcoholic steatohepatitis (NASH), which may evolve to cirrhosis later in life. ${ }^{2}$

Although no therapy has yet demonstrated efficacy with regard to NASH and fibrosis, ${ }^{1}$ docosahexaenoic acid (DHA), an omega-3 poly-unsaturated fatty acid (N3-PUFA) with anti-inflammatory and insulin-sensitizing properties, has shown promising results in the reduction of liver fat content. ${ }^{3,4}$ NAFLD has a major heritable component, especially in children. ${ }^{5,6}$ In particular, the patatin-like phospholipase domain-containing protein-3 (PNPLA3) rs738409 $\mathrm{C}>\mathrm{G}$ polymorphism, encoding for the I148M protein variant, is not only the major genetic determinant of hepatic fat content and increased liver enzymes ${ }^{7}$ but also a risk factor for NASH and fibrosis. ${ }^{8,9}$ PNPLA3 is regulated by the lipogenic program and is involved in lipid metabolism in hepatocytes, and the I148M polymorphism alters the ac-

Manuscript received 14 February 2013. Revision accepted 10 July 2013.

Address correspondence to: Valerio Nobili, Hepatometabolic Unit, Bambino Gesù Children's Hospital, Piazza S. Onofrio 4, Roma 00165, Italy, E-mail: nobili66@yahoo.it tivity of this enzyme. ${ }^{10,11}$ The I148M polymorphism influences liver damage and susceptibility to NASH early in life, ${ }^{5,9,12-15}$ synergizing with abdominal fat and carbohydrate intake. ${ }^{16,17}$ Interestingly, there is some evidence that the I148M-NAFLD association is affected by the dietary N6-PUFA/N-3-PUFA ratio, and it has been hypothesized that dietary supplementation with N-3-PUFA may reverse steatosis in carriers of the I148M polymorphism. ${ }^{18}$ The aim of this secondary analysis from a randomized controlled trial $(\mathrm{RCT})^{3,4}$ was to test whether the I148M PNPLA3 polymorphism is associated with the response to DHA supplementation in children with NAFLD.

The study protocol and the primary analysis of this RCT are described in detail elsewhere (trial identifier: NCT00885313 www.clinicaltrials.gov). ${ }^{3,4}$ Briefly, 60 children with NAFLD were randomized in equal numbers $(n=20)$ to DHA $250 \mathrm{mg} /$ day, DHA $500 \mathrm{mg} /$ day, or placebo using a parallel-arm design. Written informed consent was obtained from the parents or legal guardians of the children, and the study protocol was approved by the ethics committee of the Bambino Gesù Hospital. As reported in detail elsewhere, ${ }^{3,4}$ the primary analysis showed that DHA supplementation decreased liver steatosis and that doses of 250 and $500 \mathrm{mg} /$ day of DHA were equally effective. PNPLA3 I148 variants were assessed at the end of the RCT by means of $5^{\prime}$ nuclease Taqman assays. ${ }^{9}$ 
Table 1. Baseline Measurements of the 60 Study Children Stratified According to the I148 PNPLA3 Polymorphism

\begin{tabular}{|c|c|c|c|c|c|c|c|}
\hline & \multicolumn{2}{|c|}{$\begin{array}{c}I / I \\
\mathrm{n}=6 \\
(\mathrm{M}=5)\end{array}$} & \multicolumn{2}{|c|}{$\begin{array}{c}I / M \\
\mathrm{n}=34 \\
(\mathrm{M}=20)\end{array}$} & \multicolumn{2}{|c|}{$\begin{array}{c}M / M \\
\mathrm{n}=20 \\
(\mathrm{M}=10)\end{array}$} & \multirow[b]{2}{*}{$\mathrm{P}$ value } \\
\hline & P50 & $I Q R$ & $P 50$ & $I Q R$ & P50 & $I Q R$ & \\
\hline Age (years) & 10 & 7 & 11 & 3 & 12 & 3 & .41 \\
\hline BMI $\left(\mathrm{kg} / \mathrm{m}^{2}\right)$ & 25.5 & 4.2 & 26.0 & 4.4 & 26.7 & 2.3 & .28 \\
\hline BMI (SDS) & 2.1 & 1.2 & 1.8 & 0.7 & 1.9 & 0.6 & .34 \\
\hline Glucose (mg/dL) & 82 & 7 & 85 & 10 & 88 & 12 & .58 \\
\hline Insulin $(\mathrm{mg} / \mathrm{dL})$ & 17 & 6 & 10 & 6 & 13 & 11 & .06 \\
\hline Triglycerides $(\mathrm{mg} / \mathrm{dL})$ & 85 & 48 & 85 & 33 & 92 & 27 & .14 \\
\hline $\operatorname{ALT}(\mathrm{U} / \mathrm{L})$ & 44 & 43 & 68 & 30 & 72 & 27 & .16 \\
\hline
\end{tabular}

P50, median; IQR, interquartile range; M, males; BMI, body mass index; SDS, standard deviation score; ALT, alanine transaminase.

The present analysis evaluated the changes in liver fat, triglycerides, and alanine transaminase (ALT) using Somers' D association measure. ${ }^{19}$ Somers' D models employed for this secondary analysis had ultrasonographically determined liver fat (discrete, $0=$ none, $1=$ mild, $2=$ moderate, and $3=$ severe), triglycerides (continuous, $\mathrm{mg} / \mathrm{dL}$ ), or ALT (continuous, U/L) as outcomes and time (discrete, $0=$ baseline, $1=24$ months), DHA (discrete, $0=$ placebo, $1=250$ or $500 \mathrm{mg} /$ day), a DHA $\times$ time interaction (discrete), and PNPLA3 I148 variants (discrete, $0=\mathrm{I} / \mathrm{M}, 1=\mathrm{M} / \mathrm{M}$, $2=\mathrm{I} / \mathrm{I})$ as predictors. Repeated measures were taken into account by specifying cluster confidence intervals for each patient. The analysis was intention to treat, and there were no missing data. Statistical analysis was performed using Stata 12.1 along with the somersd package. ${ }^{20}$
The main features of the patients stratified on the basis of the PNPLA3 I148 variants are reported in Table 1. Subjects with $\mathrm{I} / \mathrm{I}, \mathrm{I} / \mathrm{M}$, and $\mathrm{M} / \mathrm{M}$ variants were similar for all characteristics. The number of I/M, M/M, and I/I polymorphisms in the DHA and placebo groups were 24 (71\%), 13 (65\%), 3 $(50 \%)$ and $10(29 \%), 7(35 \%), 3(50 \%)$. Figure 1 depicts the number of subjects with liver steatosis before and after treatment with DHA or placebo. Table 2 reports the independent contribution of PNPLA3 I148 to the effect of DHA on liver steatosis (Somers' D).

Coherently with the primary analysis, the probability of more severe steatosis after 24 months of DHA supplementation was $50 \%$ lower $(95 \%$ confidence interval [CI] -59 to $-42 \%, P<.001)$ in the combined DHA 250 and $500 \mathrm{mg} /$ day groups versus placebo. The secondary analysis, however, revealed an independent effect of PNPLA3 on the response to DHA. In fact, the probability of more severe steatosis was higher $(37 \%, 95 \%$ CI $26-48 \%, P<.001)$ for the PNPLA3 M/M versus $\mathrm{I} / \mathrm{M}$ allele and lower $(-12 \%, 95 \% \mathrm{CI}-21$ to $-3 \%, P<.05)$ for the $\mathrm{I} / \mathrm{I}$ versus $\mathrm{I} / \mathrm{M}$ allele. Expectedly, the availability on only six subjects with the I/I allele was responsible for large confidence intervals of the corresponding effect sizes. Interestingly, the higher probability of more severe steatosis at the end of the trial in the $\mathrm{M} / \mathrm{M}$ group was accompanied by a higher probability of increased triglycerides (14\%, 95\% CI 1-27\%) and ALT (14\%, 3-25\%). Even if larger numbers of subjects are needed to precisely quantify these effects, ${ }^{3,4}$ these changes are in keeping with the known effects of DHA on serum lipids.

In this secondary analysis of an RCT, ${ }^{3,4}$ we tested whether the PNPLA3 I148M variant is associated with the response to DHA supplementation in children with NAFLD. We found that the response to DHA was lower in children who were homozygous for the $148 \mathrm{M}$ and higher in those who

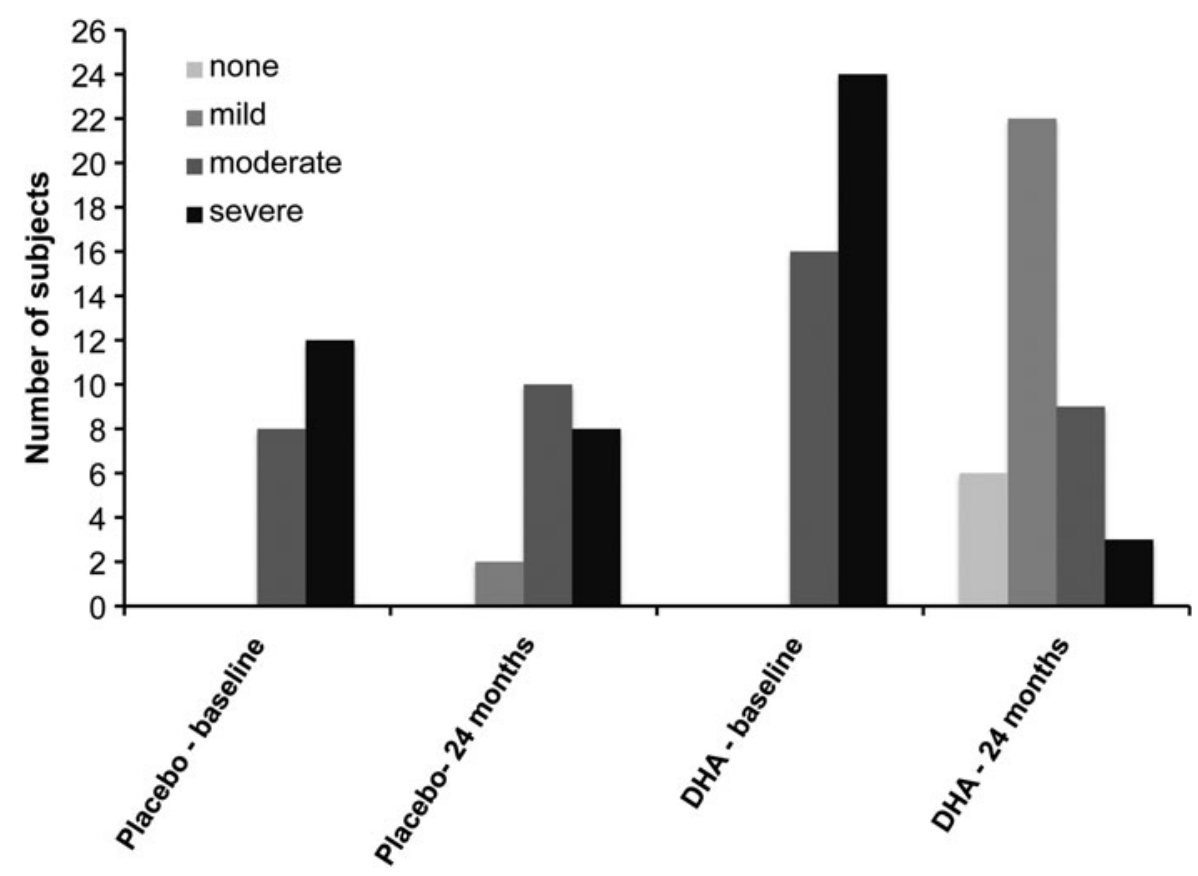

FIG. 1. Number of subjects with liver steatosis before and after treatment with docosahexaenoic acid (DHA) or placebo. 
Table 2. Independent Effect of the I148M PNPLA3 Genotype on the Response to Docosahexaenoic Acid

\begin{tabular}{lccc}
\hline & Prob. (more severe FL) & Prob. (higher TG) & Prob. (higher ALT) \\
\hline DHA versus placebo & $-.50 * * *[-0.59$ to -0.42$]$ & $-.05[-0.12$ to 0.02$]$ & $-.29 * * *[-0.38$ to -0.20$]$ \\
M/M versus I/M & $.37 * *[0.26$ to 0.48$]$ & $.14 *[0.01$ to 0.27$]$ & $.14 *[0.03$ to 0.25$]$ \\
I/I versus M/M & $-.12 *[-0.21$ to -0.03$]$ & $.01[-0.07$ to 0.09$]$ & $-.05[-0.13$ to 0.03$]$ \\
\hline
\end{tabular}

Values are probabilities with $95 \%$ confidence intervals. Effect sizes were calculated using Somers' D (see statistical analysis for details).

$* P<.05, * * * P<.001$.

Prob., probability; FL, fatty liver; TG, triglycerides; ALT, alanine aminotransferase.

were homozygous for the 148I allele as compared with heterozygotes.

The $148 \mathrm{M}$ allele was associated with $50 \%$ higher probability of more severe steatosis at the end of the trial, even if the effect of DHA on steatosis was independent from PNPLA3. The frequency of the $148 \mathrm{M}$ variant in our children was expectedly high, owing to NAFLD severity and persistently elevated ALT. ${ }^{5,8,14,15}$ To our knowledge, this is the first demonstration of a different response of NAFLD to treatment on the basis of a genetic factor. More effective treatments are needed for the subgroup of patients with NAFLD homozygous for the 148M PNPLA3 variant, because the spectrum of liver disease associated with this genetic risk factor may extend to liver cirrhosis and hepatocellular carcinoma. ${ }^{21}$

In a recent study, the dietary N-6/N-3 PUFA ratio was associated with hepatic fat content and ALT in a multiethnic sample of obese children from the United States. ${ }^{18}$ Such association was evident only in subjects who were homozygous for the 148M PNPLA3 allele, leading us to hypothesize that the $148 \mathrm{M}$ allele does not allow effective hepatic lipid remodeling in the presence of an altered dietary N-6/N-3 PUFA ratio. These data suggest that the possibility that the position of the last unsaturated bond of fatty acids may be differentially affected by the 148I and 148M PNPLA3 variants. This hypothesis needs to be addressed in further studies, as insufficient data are available on PNPLA3 affinity for N-3 PUFA. ${ }^{11}$

A reduction of dietary N-6 PUFA or alternatively, an increase of dietary N3-PUFA may be, therefore, needed to correct steatosis in subjects with the $148 \mathrm{M}$ variant. Our data are not consistent with the latter hypothesis, because DHA, a N-3 PUFA, was less effective in $148 \mathrm{M} / \mathrm{M}$ subjects. Our data are more consistent with (but, of course, do not prove) the alternative hypothesis that excessive dietary N-6 PUFA favor lipogenesis ${ }^{10,22}$ or reduce PNPLA3 hydrolytic activity or PNPLA3 contribution to lipoprotein export ${ }^{11,23,24}$ in $148 \mathrm{M}$ subjects.

The higher levels of triglycerides in $148 \mathrm{M} / \mathrm{M}$ subjects after treatment are at variance with previous results, supporting an association between the $148 \mathrm{M}$ allele and decreased levels of serum triglycerides, ${ }^{25,26}$ possibly related to impaired export of very low density lipoproteins. ${ }^{24}$ This finding may be, however, explained by the higher severity of steatosis, which is a major determinant of very low-density lipoprotein secretion, in $148 \mathrm{M} / \mathrm{M}$ subjects at the end of treatment, associated with the lower response to the effect of DHA on hepatic lipid metabolism.

Since our findings rely on an unplanned secondary analysis of an RCT, they need confirmation in specifically designed RCT, where the subjects are stratified on the basis of PNPLA3 status. However, points of strengths of the present secondary analysis are that (1) the genetic background is inherited and not susceptible to modification; (2) all patients were tested; and (3) the PNPLA3 genotypes were equally distributed among treatment arms.

In conclusion, homozygosity for the 148M PNPLA3 allele is associated with lower regression of steatosis after DHA supplementation in pediatric NAFLD. Further studies are needed to confirm our findings and to determine the effects of PNPLA3 on the changes of "hard" hepatological outcomes such as NASH and liver fibrosis.

\section{ACKNOWLEDGMENTS}

The authors thank Bruno Dalla Piccola for helpful comments and suggestions. Genetic analysis of subjects included in the study was supported by the Metabolic Liver Diseases Research Center of the Università degli Studi di Milano (University of Milan).

\section{AUTHOR DISCLOSURE STATEMENT}

All authors declare that no financial assistance was received to support the analysis and writing of this study.

\section{REFERENCES}

1. Nobili V, Svegliati-Baroni G, Alisi A, et al:: A 360-degree overview of paediatric NAFLD: recent insights. J Hepatol 2012;58:1218-1229.

2. Bugianesi E, Leone N, Vanni E, et al.: Expanding the natural history of nonalcoholic steatohepatitis: from cryptogenic cirrhosis to hepatocellular carcinoma. Gastroenterology 2002;123: 134-140.

3. Nobili V, Bedogni G, Alisi A, et al: Docosahexaenoic acid supplementation decreases liver fat content in children with non-alcoholic fatty liver disease: double-blind randomised controlled clinical trial. Arch Dis Child 2011;96:350-353.

4. Nobili V, Alisi A, Della Corte C, et al.: Docosahexaenoic acid for the treatment of fatty liver: randomised controlled trial in children. Nutr Metab Cardiovasc Dis 2012 [Epub ahead of print]; DOI: 10.1016/j.numecd.2012.10.010. 
5. Valenti L, Alisi A, Galmozzi E, et al.: I148M patatin-like phospholipase domain-containing 3 gene variant and severity of pediatric nonalcoholic fatty liver disease. Hepatology 2010;52:1274-1280.

6. Schwimmer JB, Celedon MA, Lavine JE, et al.: Heritability of nonalcoholic fatty liver disease. Gastroenterology 2009;136: 1585-1592.

7. Romeo S, Kozlitina J, Xing C, et al.: Genetic variation in PNPLA3 confers susceptibility to nonalcoholic fatty liver disease. Nat Genet 2008;40:1461-1465.

8. Sookoian S, Pirola CJ: Meta-analysis of the influence of I148M variant of patatin-like phospholipase domain containing 3 gene (PNPLA3) on the susceptibility and histological severity of nonalcoholic fatty liver disease. Hepatology 2011;53:1883-1894.

9. Valenti L, Al-Serri A, Daly AK, et al.: Homozygosity for the patatin-like phospholipase-3/adiponutrin I148M polymorphism influences liver fibrosis in patients with nonalcoholic fatty liver disease. Hepatology 2010;51:1209-1217.

10. Kumari M, Schoiswohl G, Chitraju C, et al.: Adiponutrin functions as a nutritionally regulated lysophosphatidic acid acyltransferase. Cell Metab 2012;15:691-702.

11. He S, McPhaul C, Li JZ, et al:: A sequence variation (I148M) in PNPLA3 associated with nonalcoholic fatty liver disease disrupts triglyceride hydrolysis. J Biol Chem 2010;285:6706-6715.

12. Goran MI, Walker R, Le KA, et al.: Effects of PNPLA3 on liver fat and metabolic profile in Hispanic children and adolescents. Diabetes 2010;59:3127-3130.

13. Lin YC, Chang PF, Hu FC, Yang WS, Chang MH, Ni YH: A common variant in the PNPLA3 gene is a risk factor for nonalcoholic fatty liver disease in obese Taiwanese children. $J \mathrm{Pe}$ diatr 2011;158:740-744.

14. Romeo S, Sentinelli F, Cambuli VM, et al:: The 148M allele of the PNPLA3 gene is associated with indices of liver damage early in life. J Hepatol 2010;53:335-338.

15. Santoro N, Kursawe R, D'Adamo E, et al.: A common variant in the patatin-like phospholipase 3 gene (PNPLA3) is associated with fatty liver disease in obese children and adolescents. Hepatology 2010;52:1281-1290.
16. Davis JN, Lê KA, Walker RW, et al.: Increased hepatic fat in overweight Hispanic youth influenced by interaction between genetic variation in PNPLA3 and high dietary carbohydrate and sugar consumption. Am J Clin Nutr 2010;92:1522-1527.

17. Miraglia del Giudice E, Grandone A, Cirillo G, et al.: The association of PNPLA3 variants with liver enzymes in childhood obesity is driven by the interaction with abdominal fat. PLoS ONE 2011;6:e27933.

18. Santoro N, Savoye M, Kim G, et al:: Hepatic fat accumulation is modulated by the interaction between the rs 738409 variant in the PNPLA3 gene and the dietary omega6/omega3 PUFA intake. PLOS ONE 2012;7:e37827.

19. Newson R: Parameters behind "nonparametric" statistics: Kendall's tau, Somers' D and median differences. Stat J 2002;2:45-64.

20. Newson R. SOMERSD: Stata Module to Calculate Kendall's Tau-a, Somers' D and Median Differences. http://ideas.repec.org/ c/boc/bocode/s336401.html (accessed January 2013).

21. Valenti L: PNPLA3 Ile148Met variant and hepatocellular carcinoma: a matter of fat. Dig Liver Dis 2012;44:974-975.

22. Li JZ, Huang Y, Karaman R, et al.: Chronic overexpression of PNPLA3I148M in mouse liver causes hepatic steatosis. J Clin Invest 2012;122:4130-4144.

23. Huang Y, He S, Li JZ, et al:: A feed-forward loop amplifies nutritional regulation of PNPLA3. Proc Natl Acad Sci USA 2010;107:7892-7897.

24. Pirazzi C, Adiels M, Burza MA, et al.: Patatin-like phospholipase domain-containing 3 (PNPLA3) I148M (rs738409) affects hepatic VLDL secretion in humans and in vitro. J Hepatol 2012; 57:1276-1282.

25. Palmer CN, Maglio C, Pirazzi C, et al.: Paradoxical lower serum triglyceride levels and higher type 2 diabetes mellitus susceptibility in obese individuals with the PNPLA3 148M variant. PLoS ONE 2012;7:e39362.

26. Krarup NT, Grarup N, Banasik K, et al.: The PNPLA3 rs738409 G-allele associates with reduced fasting serum triglyceride and serum cholesterol in Danes with impaired glucose regulation. PLOS ONE 2012;7:e40376. 